\title{
An analysis of cosmological perturbations in hydrodynamical and field representations
}

\author{
Júlio C. Fabris ${ }^{a}$ 凹, Sergio V.B. Gonçalves ${ }^{b}$ ] and Nazira A. Tomimura ${ }^{b}$ 阝 \\ ${ }^{a}$ Departamento de Fúsica, Universidade Federal do Espírito Santo, \\ Vitória CEP 29060-900-Espírito Santo. Brazil. \\ ${ }^{b}$ Instituto de Fúsica, Universidade Federal Fluminense \\ Niterói CEP 24210-340-Rio de Janeiro. Brazil
}

\begin{abstract}
Density fluctuations of fluids with negative pressure exhibit decreasing time behaviour in the long wavelength limit, but are strongly unstable in the small wavelength limit when a hydrodynamical approach is used. On the other hand, the corresponding gravitational waves are well behaved. We verify that the instabilities present in density fluctuations are due essentially to the hydrodynamical representation; if we turn to a field representation that lead to the same background behaviour, the instabilities are no more present. In the long wavelength limit, both approachs give the same results. We show also that this inequivalence between background and perturbative level is a feature of negative pressure fluid. When the fluid has positive pressure, the hydrodynamical representation leads to the same behaviour as the field representation both at the background and perturbative levels.
\end{abstract}

PACS number: 98.80.Bp, 95.35.+d

keywords: cosmology, perturbations theory, large scale structure.

\section{Introduction}

Fluids with negative pressures have become of utmost importance in modern cosmology. They were first considered in the context of the inflationary scenario in which the early universe has a very short period of accelerating expansion [1]. This inflationary phase solves many problems of the standard model which are connected to specific choices of

\footnotetext{
1e-mail: fabris@cce.ufes.br

2e-mail: svbg@if.uff.br

${ }^{3}$ e-mail: nazt@if.uff.br
} 
initial conditions, like the flatness and horizon problem. At the same time, the origin of the seeds of the large scale structures observed today in the universe has a natural explanation in the inflationary scenario, that considers them as quantum fluctuations of a scalar field in a de Sitter background. The inflationary phase must end up with a transition to the radiative phase of the standard model. In many aspects, the inflationary scenario is plagued with problems of arbitrary choice of parameters of the microphysics, but its great success with the above mentioned problems led the community to consider it as part of the standard scenario.

More recently, the observation of the supernova of high redshift led to the preliminary conclusion that the Universe today is in an accelerating expansion [2, 3]. This is a surprising result since there was little doubts that the Universe was desacelerating and that the desaceleration parameter $q$ was positive. If the results of the supernova observations are confirmed, the energy of the Universe today must be dominated by a fluid with negative pressure. It could be a cosmological constant, but other cases are not excluded, like a fluid of cosmic string or domain walls [4, 5, 6] or some scalar field, with a peculiar kind of potential, called quintessence [7, 8, 9, 10, 11].

In many pratical cases, these fluids with negative pressure may be represented by a perfect fluid with a barotropic equation of state $p=\alpha \rho$, with $\alpha<0$. To be more precise, in order to have an accelerating universe the strong energy condition must be violated and $\alpha<-\frac{1}{3}$. A fluid of cosmic string leads to $\alpha=-\frac{1}{3}$, representing the limiting case between an accelerating and desaccelerating universe; $\alpha=-\frac{2}{3}$ and $\alpha=-1$ represent respectively a fluid of domain wall and a cosmological constant. The quintessence fluid only approximativelly can be expressed by a barotropic fluid with $\alpha<0$.

In [12] perturbations in fluids with negative pressure were studied in the hydrodynamical representation. It was found that when the strong energy condition is violated, there are instabilities in the small wavelength limit. This result can be understood by remembering that in this limit the expansion of the universe plays no important role: the negative pressure at this limit acts in the same way as gravity and nothing can prevent the collapse. This can be easily seen in the case of a cosmic string fluid for which the density contrast behaves as $\Delta \propto t^{1 \pm \sqrt{1+\frac{n^{2}}{3}}}$. When $n \rightarrow \infty$ divergences appear, leading to the instability of the background model. In the cosmological perturbation theory, we are generally interested on the unstable modes, but that are not divergent. From here on, instabilities will refer to these undesirable divergent modes.

This study of density perturbations for a fluid of cosmic string has been extended in [13], where a two-fluid model was considered, one of them being the string fluid and the other ordinary matter with positive pressure. Special attention was payed to the case of a closed spatial section. From the point of view of the background, such models have many interesting features, mainly conected to the horizon problem and to the age of the universe. However, the study of scalar fluctuations around this background shows the presence of instability in the small scale limit, as in the case of the one fluid model. On the other hand, the study of gravitational waves for these models [14] reveals a very regular behaviour because gravitational waves are mainly connected with the scale factor behaviour, being quite insensitive to the matter representation. This indicates that the instabilities detected in [12, 13] could be due to the hydrodynamical representation, which could disappear in a more fundamental approach.

In fact, the hydrodynamic representation for fluids with negative pressure is frequently 
a very poor approximation. Negative pressures appear in situations involving phase transitions in a primordial universe (topological defects) or a fundamental self-interacting scalar field. The exact formulation involves consequently fields, and a representation using a fluid with a barotropic equation of state only in very special situations may be employed. The employment of a perfect fluid representation, mainly when fluids of negative pressure are involved, can be viewed as a practical simplification which in many situations gives the same results as those that could be obtained by employing a more fundamental field representation.

The main point is that the equivalence of a hydrodynamical representation with a more fundamental one is very restricted and it can lead to complete misleading results depending on the problem treated. The instability in the small wavelength limit quoted above is an example. Fluids with negative pressure should have a field representation where their main features could be retained. The representation we will investigate here involves the more reasonable coupling of gravity with a scalar field with a self interacting term.

The aim of this paper is to show that the scalar field representation can more conveniently retain the features we could expect from fluids with negative pressure, mainly for those that are interesting for cosmology, as the objects resulting from phase transitions, like cosmic string. We will concern mainly with the consequences of these different representations for an analysis of perturbations around a homogenous and isotropic expanding universe. We verify that when we use a field representation, with the same behaviour for the scale factor as the corresponding hydrodynamical representation, the instabilities present in the latter case are absent in the former one. This is due essentialy to the fact that when we pass from a hydrodynamical representation to a field representation of the fluid we also pass from an Euler's type equation to a Klein-Gordon's type equation, and there is no correspondence when the pressure is negative. On the other hand, for large scale perturbations, both approachs lead to the same results.

We begin by analysing a stiff matter fluid which can mimic a scalar field in a very simple way. As it is well known, a free scalar field minimally coupled to gravity reproduces the stiff matter equation of state. We will show in section 2 that for the stiff matter and free scalar field models, the agreement between the results occurs not only at the background level but also at the perturbative level. In section 3 we extend this analysis to a perfect fluid with arbitrary equation of state $p=\alpha \rho$ : we determine the corresponding field representation, showing that, at perturbative level, the equivelence between these two approachs remains only when $\alpha>0$. In section 4 we review briefly the results obtained in [13, 14 and we discuss the possibility of a field representation for this two fluid model. In section 5, we review a model of variable cosmological constant that leads, from the point of view of the evolution of the scalar factor, to the same behaviour as a cosmic string fluid. In section 6, we perform a perturbative analysis of the variable constant model, and show explicitly that they are free of instabilities, both for scalar perturbations and tensor perturbations. In section 7 , we discuss the results. 


\section{Free scalar field model}

The most simple gravity model with a scalar field is described by the action

$$
\mathcal{S}=\int \sqrt{-g}\left[R-\phi,{ }_{\alpha} \phi,{ }^{\alpha}\right] d^{4} x \quad .
$$

It represents a free scalar field minimally coupled to gravity. The field equations obtained in accordance with the principle of least action by varying $S$ with respect to the dynamical variables are:

$$
\begin{gathered}
R_{\mu \nu}-\frac{1}{2} g_{\mu \nu} R=\phi_{; \mu} \phi_{; \nu}-\frac{1}{2} g_{\mu \nu} \phi_{; \rho} \phi^{; \rho}, \\
\square \phi=0 .
\end{gathered}
$$

In a Friedmann-Robertson-Walker flat space-time (FRW), the metric describing the four dimensional geometry is

$$
d s^{2}=d t^{2}-a^{2}(t)\left(d x^{2}+d y^{2}+d z^{2}\right),
$$

and the field equations take the form

$$
\begin{aligned}
3\left(\frac{\dot{a}}{a}\right)^{2} & =\frac{1}{2} \dot{\phi}^{2}, \\
2 \frac{\ddot{a}}{a}+\left(\frac{\dot{a}}{a}\right)^{2} & =-\frac{\dot{\phi}^{2}}{2}, \\
\ddot{\phi}+3 \frac{\dot{a}}{a} \dot{\phi} & =0,
\end{aligned}
$$

where the overdot denotes the derivative with respect to the time coordinate $t$. The equations (5.6.7) are not independent due to the Bianchi identities.

On the other hand, in an universe filled with a perfect fluid we have the field equations

$$
\begin{aligned}
R_{\mu \nu}-\frac{1}{2} g_{\mu \nu} R & =8 \pi G T_{\mu \nu}, \\
T_{; \mu}^{\mu \nu} & =0
\end{aligned}
$$

where

$$
T_{\mu \nu}=(\rho+p) u_{\mu} u_{\nu}-p g_{\mu \nu},
$$

with $p=\alpha \rho, \alpha$ being a constant. The most common values of $\alpha$ of cosmological interest are 0 (pressurelless matter), $\frac{1}{3}$ (radiation) and 1 (stiff matter). Negative values of $\alpha$ has acquired increasing importance due to the inflationary paradigm and the new results coming from the supernova type Ia observations, as it was discussed before. Topological defects also require a negative equation of state. Again, equations (8,9) are connected by the Bianchi identities.

In the same FRW flat background, (8, g) take the form

$$
\begin{aligned}
3\left(\frac{\dot{a}}{a}\right)^{2} & =8 \pi G \rho, \\
2 \frac{\ddot{a}}{a}+\left(\frac{\dot{a}}{a}\right)^{2} & =-8 \pi G p, \\
\dot{\rho}+3(1+\alpha) \frac{\dot{a}}{a} \rho & =0 .
\end{aligned}
$$


It is a straightforward to check that the equations (5, 6, 77) and equations (11, 12, 13) permit the identification

$$
\rho_{\phi}=p_{\phi}=\frac{\dot{\phi}^{2}}{2}
$$

i.e., the "scalar field fluid" behaves like a stiff matter in the hydrodynamical approach. The scalar field sound velocity, in this case, is $c_{\phi}^{2}=\dot{p}_{\phi} / \dot{\rho}_{\phi}=1$. The scale factor both in the free scalar field and hydrodynamical stiff matter cases behaves as $a \propto t^{1 / 3}$.

The evaluation of the perturbed quantities follows the well known approach of Lifshitz and Khalatnikov [15, [16]. It can be treated either with the synchronous gauge or the gauge-invariant formalism. Here we choose to work in the synchronous gauge formalism, where $h_{\mu 0}=0$.

We study first density perturbations and then gravitational waves for this free field model.

\subsection{Density perturbations}

Introducing small perturbations around the background solutions, the perturbed equations for the scalar-tensor model read

$$
\begin{gathered}
\ddot{h}+2 \frac{\dot{a}}{a} \dot{h}=4 \dot{\phi} \dot{\delta \phi}, \\
\ddot{\delta \phi}+3 \frac{\dot{a}}{a} \dot{\delta} \phi+\frac{n^{2}}{a^{2}} \delta \phi-\frac{1}{2} \dot{h} \dot{\phi}=0
\end{gathered}
$$

where $h=h_{k k} / a^{2}$ and $n^{2}$ comes from the Helmholtz equation $\nabla^{2} \mathcal{Q}+n^{2} \mathcal{Q}=0$ : the scalar functions $\mathcal{Q}\left(x^{k}\right)$ are the eingefunctions of the three-dimensional Laplacian operator.

In order, to solve equations (15, 16) it is more convenient to work in the conformal time, $d t=a d \eta$. The scale factor behaves as $a \propto \eta^{1 / 2}$. In terms of this new time parameter the solution of the perturbed equations is given by [17]

$$
\delta \phi=\eta^{-3 / 2} \int \eta^{3 / 2}\left(c_{1}(n) J_{1}(n \eta)+c_{2}(n) N_{1}(n \eta)\right) d \eta
$$

where $J_{1}$ and $N_{1}$ are, respectively, the Bessel and Neumann functions of the first order, and $c_{1}(n), c_{2}(n)$ are two arbitrary constants.

We need to verify if the evolution of the perturbations are specified by the equation $\delta p_{\phi}=\alpha \delta \rho_{\phi}$ as it happens with the background evolution showed previously. This should imply that the adiabatic approximation is verified here. To do this, we consider the perturbation of the equation (2) and (10)

$$
\begin{aligned}
& \delta G^{\mu \nu}=\delta \phi,{ }^{\mu} \phi,{ }^{\nu}+\phi,{ }^{\mu} \delta \phi,{ }^{\nu}+\frac{1}{2} h^{\mu \nu} g^{\alpha \beta} \phi,_{\beta} \phi, \alpha \\
& +\frac{1}{2} g^{\mu \nu} h^{\alpha \beta} \phi,_{\beta} \phi,{ }_{\alpha}-\frac{1}{2} g^{\mu \nu} g^{\alpha \beta}\left(\delta \phi,_{\beta} \phi,_{\alpha}+\phi,{ }_{\beta} \delta \phi, \alpha\right) \quad, \\
& \delta T^{\mu \nu}=\left(\delta \rho_{\phi}+\delta p_{\phi}\right) U^{\mu} U^{\nu}+\left(\rho_{\phi}+p_{\phi}\right)\left(\delta U^{\mu} U^{\nu}+U^{\mu} \delta U^{\nu}\right)-\delta p_{\phi} g^{\mu \nu}+p_{\phi} h^{\mu \nu} .
\end{aligned}
$$

Using the synchronous gauge condition, we have

$$
\delta G_{00}=\dot{\phi} \delta \dot{\phi}
$$




$$
\begin{gathered}
\delta G_{i j}=-\frac{1}{2} h_{i j} \dot{\phi}^{2}+a^{2} \delta_{i j} \dot{\phi} \delta \dot{\phi} \\
\delta T_{00}=\delta \rho_{\phi} \\
\delta T_{i j}=-p_{\phi} h_{i j}+a^{2} \delta_{i j} \delta p_{\phi}
\end{gathered}
$$

If we consider the perturbed Einstein equations $\delta G_{\mu \nu}=8 \pi G \delta T_{\mu \nu}$ and the values of $\rho_{\phi}$ and $p_{\phi}$ obtained from equations (11) and (12), we have that

$$
\delta \rho_{\phi}=\delta p_{\phi}
$$

In the hydrodynamical approach, the solution for the Einstein perturbed equations with $p=\rho$ and $\delta p=\delta \rho$, leads to the expression [12]

$$
\Delta=\frac{\delta \rho}{\rho}=\eta^{-3 / 2} \int \eta^{5 / 2}\left(d_{1}(n) J_{0}(n \eta)+d_{2}(n) N_{0}(n \eta)\right) d \eta
$$

where $d_{1}(n)$ and $d_{2}(n)$ are the integration constants. Remembering that $\rho_{\phi}=\frac{\phi^{\prime 2}}{2 a^{2}}$ and $\delta \rho_{\phi}=2 \frac{\phi^{\prime} \delta \phi^{\prime}}{a^{2}}$, the quantity $\Delta_{\phi}=\frac{\delta \phi^{\prime}}{\phi^{\prime}}$, computed from (17) reduces to (25), using simple recurrence relations for Bessel's functions.

Hence, in this simple model where the matter is described by a scalar field, the background and perturbed equations can be put in a barotropic form. The "velocity of sound" $\delta p_{\phi} / \delta \rho_{\phi}$ is the same as the one defined by $c_{\phi}^{2}=\dot{p}_{\phi} / \dot{\rho}_{\phi}$ in agreement with [18], where it is shown that this result corresponds to the low-frequency regime of the scalar field oscillations.

\subsection{Gravitational waves}

Here, the perturbed equation of gravitational waves is:

$$
h^{\prime \prime}-2 \frac{a^{\prime}}{a} h^{\prime}+\left[n^{2}-2 \frac{a^{\prime \prime}}{a}+2 \frac{a^{\prime 2}}{a^{2}}\right] h=0,
$$

where $h_{i j}=h(\eta) Q_{i j}, Q_{i j}$ being a tracelless transverse eigenfunction on the spatial section, and primes denote derivative with respect to conformal time $d \eta=a d t$.

After inserting the backgroung solutions, we obtain the solution of the equation (26), as follow:

$$
h=\eta\left(e_{1}(n) J_{1}(n \eta)+e_{2} N_{1}(n \eta)\right)
$$

where $e_{1}(n)$ and $e_{2}(n)$ are the integration constants. This solution is valid for both representations.

It is easy to verify that the above solution is well-behaved and stable.

\section{Field representation for an arbitrary barotropic equa- tion of state}

Let us return to the Einstein's equations coupled to a perfect fluid, with a barotropic equation of state $p=\alpha \rho$. Solving the Einstein's equation for a flat spatial section, we 
obtain for the scale factor $a=a_{0} t^{\frac{2}{3(1+\alpha)}}$. Let us now consider a self interacting scalar field coupled to gravity. The Lagrangian has the form,

$$
L=\sqrt{-g}\left[R-\phi_{; \rho} \phi^{; \rho}+2 V(\phi)\right]
$$

where the potential $V(\phi)$ represents the self interaction term. The field equations are

$$
R_{\mu \nu}-\frac{1}{2} g_{\mu \nu} R=\phi_{; \mu} \phi_{; \nu}-\frac{1}{2} g_{\mu \nu} \phi_{; \rho} \phi^{; \rho}+g_{\mu \nu} V(\phi) \quad, \quad \square \phi=-V_{\phi}(\phi) \quad,
$$

where $V_{\phi}$ means partial derivative of the potential with respect to $\phi$.

This scalar-tensor model may lead to the same behaviour of the scale factor as in the perfect fluid model provided that the potential takes the form,

$$
V(\phi)=\frac{2}{3} \frac{(1-\alpha)}{(1+\alpha)^{2}} \exp (\mp \sqrt{3(1+\alpha)} \phi)
$$

Indeed, for a FRW background, this potential leads to the solutions

$$
a=a_{0} t^{\frac{2}{3(1+\alpha)}} \quad, \quad \phi= \pm \frac{2}{\sqrt{3(1+\alpha)}} \ln t .
$$

For the case of a cosmic string fluid, $a \propto t$, the potential is $V(\phi)=-2 e^{-\sqrt{2} \phi}$.

We now turn to the perturbative level. The perturbation of the scalar-tensor model leads to

$$
\begin{aligned}
h^{\prime \prime}+\frac{a^{\prime}}{a} h^{\prime} & =4 \phi^{\prime} \delta \phi^{\prime}+2\left(\phi^{\prime \prime}+2 \frac{a^{\prime}}{a} \phi^{\prime}\right) \delta \phi, \\
\delta \phi^{\prime \prime}+2 \frac{a^{\prime}}{a} \delta^{\prime}+\left(n^{2}+V_{\phi \phi} a^{2}\right) \delta \phi & =\frac{1}{2} \phi^{\prime} h^{\prime},
\end{aligned}
$$

where we follow the same definitions as before and we have employed the conformal time.

Combining equations (32, 33), and inserting the background solutions, we obtain the third order differential equation

$$
\begin{aligned}
\delta \phi^{\prime \prime \prime} & +\frac{7+3 \alpha}{1+3 \alpha} \frac{\delta \phi^{\prime \prime}}{\eta}+\left\{n^{2}+\left[\frac{2-24 \alpha-18 \alpha^{2}}{(1+3 \alpha)^{2} \eta^{2}}\right]\right\} \delta \phi^{\prime} \\
& +\left\{3 \frac{1+\alpha}{1+3 \alpha} \frac{n^{2}}{\eta}-18 \frac{1-\alpha^{2}}{(1+3 \alpha)^{2}} \frac{1}{\eta^{3}}\right\} \delta \phi=0 .
\end{aligned}
$$

One solution for this equation is $\delta \phi=\eta^{-3 \frac{1+\alpha}{1+3 \alpha}}$, which is given by the residual coordinate transformation freedom characteristic of the synchronous coordinate condition. Using this known solution and some suitable transformation, we can reduce the third order equation to a second order Bessel equation. The final solution for $\delta \phi$ is

$$
\delta \phi=\eta^{-3 \frac{1+\alpha}{1+3 \alpha}} \int c_{ \pm} \eta^{\frac{3}{2}} J_{ \pm \nu}(n \eta) d \eta
$$

where $c_{ \pm}$are integration constants, that in general depend on $n$, and $J_{ \pm \nu}$ is a Bessel function of order $\nu=\frac{5+3 \alpha}{2(1+3 \alpha)}$. 
The solution for the density contrast in the case of the hydrodynamical representation, with $p=\alpha \rho$ is well known[12]. It can be written as

$$
\begin{aligned}
& \Delta=\eta^{-3 \frac{1+\alpha}{1+3 \alpha}} \int \eta^{\frac{5}{2}}\left(c_{+} J_{\mu}(\sqrt{\alpha} n \eta)+c_{-} J_{-\mu}(\sqrt{\alpha} n \eta)\right) d \eta \quad, \alpha>0 \\
& \Delta=\eta^{-3 \frac{1+\alpha}{1+3 \alpha}} \int \eta^{\frac{5}{2}}\left(c_{+} I_{\mu}(\sqrt{-\alpha} n \eta)+c_{-} K_{\mu}(\sqrt{-\alpha} n \eta)\right) d \eta \quad, \quad \alpha<0
\end{aligned}
$$

where now $I_{\mu}$ and $K_{\mu}$ are the modified Bessel functions, and $\mu=\frac{3}{2}\left(\frac{1-\alpha}{1+\alpha}\right)$. In order to connect both results we must remember that $\Delta_{\phi}=\frac{\delta \rho_{\phi}}{\rho_{\phi}}$, where $\rho_{\phi}=\frac{\dot{\phi}^{2}}{2}+V(\phi)$. Using some Bessel's recurrence relations, as in the previous section we find

$$
\Delta_{\phi}=C_{ \pm} \eta^{-3 \frac{1+\alpha}{1+3 \alpha}}\left\{\left(1-\frac{1}{\alpha}\right) \eta^{\frac{5}{2}} J_{ \pm \mu}(n \eta)+\int \eta^{\frac{5}{2}} J_{ \pm \mu}(n \eta) d \eta\right\}
$$

This expression differs from (36) by the factor $\alpha$ in the argument of the Bessel function, and by the first term in (38). However, when $\alpha>0$ solutions (36, 38) have the same behaviour in the large and small wavelength limit. For $\alpha=1$, the equivalence between the two approaches is complete, as can be easily checked by comparing (38) with (36) for this special case. For $\alpha<0$, the correspondence between solutions (37,38) occurs only for $n \rightarrow$ 0 (large scale perturbations); for $n \rightarrow \infty$ (small scale perturbations) the hydrodynamical representation exhibits strong instabilities while the scalar field representation displays accoustic oscillation.

Finally we remark that both representations give the same behaviour for gravitational waves.

\section{Perturbations in cosmic string fluid}

An important particular case of the scalar-tensor model develloped in the previous section concerns the case of cosmic string. A cosmic string fluid may mimic a perfect fluid with an equation of state $p=-\frac{\rho}{3}$. In [12] the fate of density perturbation in fluids with negative pressure has been studied. The main conclusion was that, in the long wavelength approximation, there are only decreasing modes when the equation of state is such that the strong energy condition is violated; for small wavelengths, instabilities can arise due to the fact that the pressure contributes in the same sense as gravity. For the equation of state of a cosmic string fluid displayed above, density perturbations behave as

$$
\Delta=t^{-1 \pm \sqrt{1+\frac{n^{2}}{3}}}
$$

Hence, in the small wavelength limit, $n \rightarrow \infty$, instabilities come to scene.

A scalar-tensor representation of the cosmic string fluid is given by a scalar tensor model with $V(\phi)=2 \exp ( \pm \sqrt{2} \phi)$. A perturbation analysis as it was performed in section 3 leads to the following expression for the perturbation in the scalar field (the integration procedure follows very closely to that sketched in the previous section):

$$
\Delta_{\phi} \propto t^{-1 \pm \sqrt{1-n^{2}}}
$$


In the large wavelength limit both representations give the same results; in the small wavelength limit the hydrodynamical representation display instabilities while the scalartensor model exhibits accoustic oscillations.

In 13] it was considered the case of two non interacting fluid, one of them represented by an equation of state like $p=-(1 / 3) \rho$ while the second fluid is the ordinary matter with a barotropic equation of state $p=\alpha \rho$. This may represent in more realistic way the presence of fluids with negative pressure in the universe. The equations of motion are

$$
\begin{gathered}
3\left(\frac{\dot{a}}{a}\right)^{2}+3 \frac{k}{a^{2}}=8 \pi G\left(\rho_{m}+\rho_{s}\right), \\
2 \frac{\ddot{a}}{a}+\left(\frac{\dot{a}}{a}\right)^{2}+\frac{k}{a^{2}}=\frac{8 \pi G}{3}\left(\rho_{s}-3 \alpha \rho_{m}\right), \\
\dot{\rho}_{m}+3 \frac{\dot{a}}{a}(1+\alpha) \rho_{m}=0, \\
\dot{\rho}_{s}+2 \frac{\dot{a}}{a} \rho_{s}=0 .
\end{gathered}
$$

In this equations $\rho_{m}$ and $\rho_{s}$ denote the ordinary fluid and stringlike fluid densities respectivelly.

The background solutions are:

$$
\begin{aligned}
& a=a_{0} \sinh ^{2}\left(\frac{\sqrt{\gamma}}{2} \eta\right), \text { when } \quad \alpha=0 ; \\
& a=a_{0} \sinh (\sqrt{\gamma} \eta), \text { when } \quad \alpha=1 / 3 ; \\
& a=a_{0} \sqrt{\sinh (2 \sqrt{\gamma} \eta)}, \text { when } \quad \alpha=1,
\end{aligned}
$$

where $\gamma=\left|\frac{8}{3} \pi G \rho_{0 s}-k\right|, \rho_{0 s}$ is defined as the ratio $\rho_{s}=\frac{\rho_{0 s}}{a^{2}}, \eta$ being the conformal time.

\subsection{Density perturbations}

Perturbing the Einstein's equations and imposing the syncrhonous coordinate condition, a set of equations for the ordinary matter and the cosmic fluid perturbations is obtained:

$$
\begin{gathered}
\ddot{h}+2 \frac{\dot{a}}{a} \dot{h}=-6 \frac{\ddot{a}}{a} \Delta_{m}, \\
\dot{\Delta}_{m}+(1+\alpha)\left(\Psi-\frac{\dot{h}}{2}\right)=0, \\
(1+\alpha) \dot{\Psi}+(1+\alpha)(2-3 \alpha) \frac{\dot{a}}{a} \Psi-\frac{n^{2}}{a^{2}} \alpha \Delta_{m}=0, \\
\dot{\Delta}_{s}+\frac{2}{3}\left(\Theta-\frac{\dot{h}}{2}\right)=0, \\
\dot{\Theta}+3 \frac{\dot{a}}{a} \Theta+\frac{n^{2}}{2 a^{2}} \Delta_{s}=0 .
\end{gathered}
$$

The integration procedure is standard [13], so we will just present the final results: 


\begin{tabular}{|c||c|c|c|}
\hline model & perturbations & material phase $(t \rightarrow 0)$ & string phase $(t \rightarrow \infty)$ \\
\hline \hline \multirow{2}{*}{$\alpha=0$} & $\Delta_{m}$ & $\eta^{2}$ & constant \\
\cline { 2 - 4 } & $\Delta_{s}$ & $\frac{1}{\eta^{\frac{2}{3}}}\left(a_{1} K_{\frac{3}{2}}(n \eta)+a_{2} I_{\frac{3}{2}}(n \eta)\right)$ & $t^{-1 \pm \sqrt{1+\frac{n^{2}}{3}}}$ \\
\hline \multirow{2}{*}{$\alpha=1 / 3$} & $\Delta_{m}$ & $\left.\frac{1}{\eta^{\frac{1}{2}} \int \eta^{\frac{5}{2}}} c_{1} J_{\frac{1}{2}}(n \eta)+c_{2} J_{-\frac{1}{2}}(n \eta)\right] d \eta$ & $\cos \left(\frac{n}{\sqrt{3}} \ln t\right)$ \\
\cline { 2 - 4 } & $\Delta_{s}$ & $\frac{1}{\eta^{\frac{1}{2}}}\left(d_{1} I_{\frac{1}{2}}(n \eta)+d_{2} K_{\frac{1}{2}}(n \eta)\right)$ & $t^{-1 \pm \sqrt{1+\frac{n^{2}}{3}}}$ \\
\hline \multirow{2}{*}{$\alpha=1$} & $\Delta_{m}$ & $\frac{1}{\eta^{\frac{3}{2}} \int \eta^{\frac{5}{2}}} c_{1} J_{0}(n \eta)+c_{2} J_{0}(n \eta) d \eta$ & $\cos (n \ln t)$ \\
\cline { 2 - 4 } & $\Delta_{s}$ & $\left.d_{1} I_{0}(n \eta)+d_{2} K_{0}(n \eta)\right)$ & $t^{-1 \pm \sqrt{1+\frac{n^{2}}{3}}}$ \\
\hline
\end{tabular}

We must exhibit the behaviour of the perturbative model for $t \rightarrow 0$ and $t \rightarrow \infty$ to find an instability in the density perturbations of cosmic string fluid. Indeed, for small wavelengths, i.e. $n \rightarrow \infty$, the modified Bessel functions $I_{\nu}(x)$ and $K_{\nu}(x)$ go as $e^{ \pm x} / \sqrt{x}$. However, the ordinary matter has a very regular behaviour for $t \rightarrow 0$ and $t \rightarrow \infty$.

The instabilities presented above can be attributed to the matter content of the universe and, mainly, to the approach used to describe the density perturbations. We will see that these instabilities do not appear in gravitational waves because the matter content of the universe plays a less important role, being only sensitive to the behaviour of scale factor.

In fact, the same behaviour for the scale factor described above can be obtained by a scalar-tensor model, with a suitable potential, coupled to ordinary matter. For example, in the case of pressurelles ordinary matter $(p=0)$, the results for the background can be recovered if the potential reads

$$
V(\phi)=V_{0} \sinh ^{-4}(C \phi) \quad, \quad V_{0}=\frac{8 \Omega^{2}+2}{a_{0}^{2}} \quad, \quad C=\frac{1}{\sqrt{8 \Omega^{2}+2}} \quad, \quad \Omega=\frac{2 \pi G \rho_{0}}{3},
$$

where $\rho_{0}$ is defined by the first integral of the conservation of the energy-moment tensor for the pressurelless fluid, $\rho=\frac{\rho_{0}}{a^{3}}$. We note that the scalar field representation for the two fluid model (pressurelles matter plus string fluid) requires a different potential with respect to the case of the one fluid model (only string fluid).

At the perturbative level, the effect of replacing the barotropic fluid with negative pressure by a scalar-field model is quite the same as that described in section 3. With respect with the preceding table of solutions, the modified Bessel functions must be replaced by ordinary Bessel functions for the perturbation in the exotic fluid. Hence the instability present in the small wavelength limit disappear again; the behaviour in the long wavelength limit is not changed.

\subsection{Gravitational waves}

Following the work [14], we have the perturbed equations that govern the evolution of gravitational waves

$$
h^{\prime \prime}-2 \frac{a^{\prime}}{a} h^{\prime}+\left[\tilde{n}^{2}-2\left(\frac{a^{\prime \prime}}{a}-\frac{a^{2}}{a^{2}}\right)\right]=0
$$

where $\tilde{n}^{2}=\left(1 / r^{2}\right)\left(n^{2}+2 k\right)$ and prime here denotes derivative with respect to $\theta=r \eta$. 
After inserting the background solutions (45,46, 47), equation (54) can be generally rewritten in terms of a hypergeometric equation. Its final solutions for different phases of the evolution of the universe read as follows:

\begin{tabular}{|c|c|c|}
\hline model & $h_{1}$ & $h_{2}$ \\
\hline$\alpha=-1$ & $\begin{array}{c}\quad \sqrt{x^{2}-1}\left[\frac{1+x}{2}\right]^{-2+\sqrt{1-\tilde{n}^{2}}} \times \\
{ }_{2} F_{1}\left(2-\sqrt{1-\tilde{n}^{2}}, \frac{1}{2}-\sqrt{1-\tilde{n}^{2}}\right. \\
\left.1-2 \sqrt{1-\tilde{n}^{2}}, \frac{2}{1+x}\right)\end{array}$ & $\begin{array}{c}\sqrt{x^{2}-1}\left[\frac{1+x}{2}\right]^{-2-\sqrt{1-\tilde{n}^{2}}} \times \\
{ }_{2} F_{1}\left(\frac{1}{2}+\sqrt{1-\tilde{n}^{2}}, 2+\sqrt{1-\tilde{n}^{2}}\right. \\
\left.1+2 \sqrt{1-\tilde{n}^{2}}, \frac{2}{1+x}\right)\end{array}$ \\
\hline$\alpha=1 / 3$ & $\exp \left(\left(\sqrt{1-\tilde{n}^{2}}\right) \eta\right) \sinh \eta$ & $\exp \left(-\left(\sqrt{1-\tilde{n}^{2}}\right) \eta\right) \sinh \eta$ \\
\hline$\alpha=0$ & $\begin{array}{c}\sqrt{x^{2}-1}\left[\frac{1+x}{2}\right]^{1+\sqrt{4-\tilde{n}^{2}}} \times \\
{ }_{2} F_{1}\left(-1-\sqrt{4-\tilde{n}^{2}}, \frac{1}{2}-\sqrt{4-\tilde{n}^{2}}\right. \\
\left.1-2 \sqrt{4-\tilde{n}^{2}}, \frac{2}{1+x}\right)\end{array}$ & $\begin{array}{c}\sqrt{x^{2}-1}\left[\frac{1+x}{2}\right]^{1-\sqrt{4-\tilde{n}^{2}}} \times \\
{ }_{2} F_{1}\left(\frac{1}{2}+\sqrt{4-\tilde{n}^{2}},-1+\sqrt{4-\tilde{n}^{2}}\right. \\
\left.1+2 \sqrt{4-\tilde{n}^{2}}, \frac{2}{1+x}\right)\end{array}$ \\
\hline$\alpha=1$ & $\begin{array}{c}\sqrt{x^{2}-1}\left[\frac{1+x}{2}\right]^{\left(-1+\sqrt{\left.1-4 \tilde{n}^{2}\right) / 2}\right.} \times \\
{ }_{2} F_{1}\left(\frac{1-\sqrt{1-4 \tilde{n}^{2}}}{2}, \frac{1-\sqrt{1-4 \tilde{n}^{2}}}{2},\right. \\
\left.1-\sqrt{1-4 \tilde{n}^{2}}, \frac{2}{1+x}\right)\end{array}$ & $\begin{array}{l}\sqrt{x^{2}-1}\left[\frac{1+x}{2}\right]^{\left(-1-\sqrt{1-4 \tilde{n}^{2}}\right) / 2} \times \\
\times \quad{ }_{2} F_{1}\left(\frac{1+\sqrt{1-4 \tilde{n}^{2}}}{2}, \frac{1+\sqrt{1-4 \tilde{n}^{2}}}{2},\right. \\
\left.1+\sqrt{1-4 \tilde{n}^{2}}, \frac{2}{1+x}\right)\end{array}$ \\
\hline
\end{tabular}

where in the above expressions, ${ }_{2} F_{1}$ is a hypergeometric function and $x=\cos (r \theta), r$ being a constant.

In this two-fluid model, the behaviour of gravitational waves in a closed universe exhibits, in what concerns the behaviour of the scale factor, the dynamic of an open universe. It would cause, also, distorsion in the spectrum of the CMBR. The determination of the evolution of perturbations and its connection with this distorsion is technically difficult to be evaluated. But, for the proposal of the present work, the fundamental feature to be retained in the above solutions is that they do not exhibit instabilities.

\section{Time dependent cosmological constant model}

We present for completeness the background equations based on the reference [19] which is the traditional scalar-tensor theory with a potential that is equivalent to a time dependent cosmological constant model. This is one of the cases which can be represented phenomenologically by a fluid with an equation of state $p=-\frac{\rho}{3}$ in what concerns the behaviour of the scale factor. The action is given by

$$
\mathcal{S}=\frac{1}{16 \pi G} \int \sqrt{-g}\left[\phi R-\phi^{-1} \omega g^{\mu \nu} \partial_{\mu} \partial_{\nu} \phi+2 \phi \Lambda(\phi)\right] d^{4} x+\mathcal{S}_{n g}
$$

We remark, however, that in the present case we have a non-minimally coupled scalar field, in opposition to the models described before.

The field equations are

$$
G_{\mu \nu}=\frac{8 \pi T_{\mu \nu}}{\phi}+\Lambda(\phi) g_{\mu \nu}+\omega \phi^{-2}\left(\phi, \mu \phi,_{\nu}-\frac{1}{2} g_{\mu \nu} \phi,_{\alpha} \phi,^{\alpha}\right)+\phi^{-1}\left(\phi ;_{\mu \nu}-g_{\mu \nu} \square \phi\right)
$$




$$
\square \phi+\frac{2 \phi^{2} d \Lambda / d \phi-2 \phi \Lambda(\phi)}{3+2 \omega}=\frac{1}{3+2 \omega}\left(8 \pi T-\frac{d \omega}{d \phi} \phi_{, \alpha} \phi^{\alpha}\right) .
$$

We shall consider the case where $\omega=$ constant, $\Lambda(\phi)=c \phi^{m}$ and $\phi=\phi_{1} t^{q}$, with $c, m$ and $q$ constants. The ansatz allow us to obtain analytical solutions in the form of power law, which leads to closed expressions for the perturbative equations.

The energy-momentum tensor describes an ordinary perfect fluid, such that

$$
T^{\mu \nu}{ }_{\nu}=0 \quad .
$$

The equations of motion are

$$
\begin{gathered}
3 \frac{\dot{a}^{2}}{a^{2}}+3 \frac{k}{a^{2}}-c \phi^{m}=\frac{8 \pi \rho}{\phi}+\frac{\omega}{2} \frac{\dot{\phi}^{2}}{\phi^{2}}-3 \frac{\dot{a} \frac{\dot{\phi}}{a}}{\phi}, \\
-2 \frac{\ddot{a}}{a}-\frac{\dot{a}^{2}}{a^{2}}-\frac{k}{a^{2}}+c \phi^{m}=\frac{8 \pi p}{\phi}+\frac{\omega}{2} \frac{\dot{\phi}^{2}}{\phi^{2}}+\frac{\ddot{\phi}}{\phi}+2 \frac{\dot{a}}{a} \frac{\dot{\phi}}{\phi}, \\
\frac{\ddot{\phi}}{\phi}+3 \frac{\dot{a}}{a} \frac{\dot{\phi}}{\phi}=\frac{2 c(1-m) \phi^{m}}{3+2 \omega}+\frac{8 \pi(\rho-3 p)}{\phi(3+2 \omega)} .
\end{gathered}
$$

The background solutions are

\begin{tabular}{|c||c|c|c|c|c|}
\hline model & curvature & $a(t)$ & $\phi(t)$ & $\rho$ & $\Lambda(t)$ \\
\hline \hline \multirow{2}{*}{$\rho=0$} & $k \neq 0$ & $a_{1} t$ & $\phi_{1} t^{\frac{-2}{m}}$ & - & $\Lambda_{1} / t^{2}$ \\
\cline { 2 - 6 } & $k=0$ & $a_{1} t$ & $\phi_{1} t^{\frac{-2}{1+\sqrt{3+2 \omega}}}$ & - & $\Lambda_{1} / t^{2}$ \\
\hline$p=\alpha \rho$ & any $k$ & $a_{1} t$ & $\phi_{1} t^{-(1+3 \alpha)}$ & $s / a^{3(1+\alpha)}$ & $\Lambda_{1} / t^{2}$ \\
\hline$p=-\rho$ & any $k$ & $a_{1} t$ & $\phi_{1} t^{2}$ & $\rho_{0}=$ const. & $\Lambda_{1} / t^{2}$ \\
\hline
\end{tabular}

where $a_{1}, \phi_{1}, \Lambda_{1}$, and $s$ are constants.

The solutions for the scale factor above are characteristic of an equation of state $p_{\phi}=-\frac{1}{3} \rho_{\phi}$.

We remark that in all above solutions the scale-factor behaves as $a \propto t$ corresponding to a "coasting" universe. From the point of view of the background behaviour, the above solutions exhibit the same characteristics as the perfect fluid formulation.

\section{Perturbations in a time dependent cosmological constant model}

We extend our perturbative analysis for this model, first for density perturbations and then for gravitational waves. 


\subsection{Density perturbations}

The perturbed equations for the time dependent cosmological constant model are:

$$
\begin{gathered}
\ddot{h}+2 \frac{\dot{a}}{a} \dot{h}=\frac{16 \pi}{\phi}(\Delta-\lambda)\left(\frac{3 \alpha \omega+3 \alpha+\omega+2}{3+2 \omega}\right) \rho \\
-2 c m \phi^{m}\left(\frac{m+2 \omega+2}{3+2 \omega}\right) \lambda+2 \ddot{\lambda}+4 \frac{\dot{\phi}}{\phi}(1+\omega) \dot{\lambda}, \\
\ddot{\lambda}+\left(2 \frac{\dot{\phi}}{\phi}+3 \frac{\dot{a}}{a}\right) \dot{\lambda}+\left(\frac{\ddot{\phi}}{\phi}+3 \frac{\dot{a}}{a} \frac{\dot{\phi}}{\phi}\right) \lambda-\frac{1}{2} \frac{\dot{\phi}}{\phi} \dot{h} \\
+\frac{n^{2}}{a^{2}} \lambda+\frac{2 c\left(m^{2}-1\right) \phi^{m}}{3+2 \omega} \lambda=\frac{8 \pi}{(3+2 \omega) \phi} \Delta(1-3 \alpha) \rho \\
\frac{\partial}{\partial t}\left(a^{5} \delta U^{k}(1+\alpha) \rho\right)=-a^{3} \alpha \rho \Delta,{ }^{k},
\end{gathered}
$$

where $h=h_{k k} a^{-2}, \lambda=\delta \phi / \phi$ and $\Delta=\delta \rho / \rho$. All functions are spatially expanded into spherical harmonics $f(x, t)=f(t) \mathcal{Q}$, with $\nabla^{2} \mathcal{Q}=-n^{2} \mathcal{Q}$.

Next, we solve the above equations for the vacuum fluid $(\alpha=-1)$, the empty universe $(\rho=0)$, radiation phase $(\alpha=1 / 3)$, and dust phase $(\alpha=0)$. We use the background relations in order to simplify the final system of equations.

\subsubsection{Vacuum fluid phase $(\alpha=-1)$}

In this case $\Delta=0$ and we have the following equations:

$$
\begin{gathered}
\ddot{h}+\frac{2}{t} \dot{h}=(1+2 \omega) \frac{\lambda}{t^{2}}+8(1+\omega) \frac{\dot{\lambda}}{t}+2 \ddot{\lambda}, \\
\ddot{\lambda}+7 \frac{\dot{\lambda}}{t}+\left[8+\frac{n^{2}}{a_{1}}\right] \frac{\lambda}{t^{2}}-\frac{1}{t} \dot{h}=0,
\end{gathered}
$$

with the solution

$$
\lambda=t^{p} \quad \text { and } \quad h \propto t^{p} \quad, \quad \text { where } \quad p=-2 \pm \sqrt{8 \omega-\frac{n^{2}}{a_{1}^{2}}} .
$$

There is no divergent behaviour in the small wavelength limit.

\subsubsection{Empty universe $(\rho=0)$}

Here, the system of second order differential equations is

$$
\begin{gathered}
\ddot{h}+\frac{2}{t} \dot{h}=-\frac{4}{m}\left[\frac{m+2 \omega+2}{3+2 \omega}\right] \frac{\lambda}{t^{2}}-\frac{8(1+\omega)}{m} \frac{\dot{\lambda}}{t}+2 \ddot{\lambda}, \\
\ddot{\lambda}+\left[\frac{3 m-4}{m}\right] \dot{\lambda}+\left[\frac{n^{2}}{a_{1}^{2}}-4(2+m) \frac{1-m}{m^{2}}\right] \frac{\lambda}{t^{2}}+\frac{1}{m} \frac{\dot{h}}{t}=0,
\end{gathered}
$$


whose solution is

$$
\lambda=t^{p} \quad \text { and } \quad h \propto t^{p} \quad \text { where } \quad p=\frac{1}{m}\left(1-m \pm \sqrt{9+6 m-3 m^{2}+8 \omega-m^{2} \frac{n^{2}}{a_{1}^{2}}}\right)
$$

where $m$ is arbitrary for $k \neq 0$ and $m=1 \pm \sqrt{3+2 \omega}$ for $k=0$. As in the preceding case, the solutions are stable.

\subsubsection{Radiation phase $(\alpha=1 / 3)$}

Combining conveniently the perturbed equations, we find a fifth order Euler's type equation for $\lambda$ :

$$
\begin{array}{r}
\lambda^{v}+11 \frac{\lambda^{i v}}{t}+\left[\frac{4}{3} \frac{n^{2}}{a_{1}^{2}}+31-2 d-2 \frac{k}{a_{1}^{2}}\right] \frac{\dddot{\lambda}}{t^{2}}+\left[\frac{16}{3} \frac{n^{2}}{a_{1}^{2}}+22-8 d-8 \frac{k}{a_{1}^{2}}\right] \frac{\ddot{\lambda}}{t^{3}} \\
+\left[\frac{1}{3} \frac{n^{4}}{a_{1}^{4}}+\frac{n^{2}}{a_{1}^{2}}\left(\frac{8}{3}+\frac{2}{3} d-2 \frac{k}{a_{1}^{2}}\right)+2-4 d-4 \frac{k}{a_{1}^{2}}\right] \frac{\dot{\lambda}}{t^{4}}+\left[\frac{1}{3} \frac{n^{4}}{a_{1}^{4}}+\frac{n^{2}}{a_{1}^{2}}\left(\frac{2}{3} d-2 \frac{k}{a_{1}^{2}}\right)\right] \frac{\lambda}{t^{5}}=0
\end{array}
$$

with $d=3+2 \omega$. Due to the residual coordinate transformation freedom characteristic of the synchronous coordinate condition, $\lambda \propto t^{-1}$ is a solution of this equation. Hence, we can reduce it to a fourth order equation. Supposing a solution of the type $\lambda \propto t^{r}$, we find the polynomial equation for $r$

$$
r^{4}+\left(\frac{4}{3} \frac{n^{2}}{a_{1}^{2}}-2 d-2 \frac{k}{a_{1}^{2}}\right) r^{2}+\left(\frac{1}{3} \frac{n^{4}}{a_{1}^{4}}-\frac{n^{2}}{a_{1}^{2}}\left(-\frac{2}{3} d+2 \frac{k}{a_{1}^{2}}\right)\right)=0
$$

whose solutions are

$$
r_{ \pm}^{2}=-\frac{2}{3} \frac{n^{2}}{a_{1}^{2}}+d+\frac{k}{a_{1}^{2}} \pm \sqrt{\frac{n^{4}}{9 a_{1}^{4}}+\left(\frac{2}{3} \frac{k}{a_{1}^{2}}-2 d\right) \frac{n^{2}}{a_{1}^{2}}+\left(d+\frac{k}{a_{1}^{2}}\right)^{2}} .
$$

In the small wavelength limit, these solutions display an oscillatory behaviour, hence stability. In the longwavelength limit, this expression reduces to $r_{ \pm}= \pm \sqrt{2\left(d+\frac{k}{a_{1}^{2}}\right)}$.

\subsubsection{Dust Phase $(\alpha=0)$}

In this case, the equations governing the evolution of density perturbations are

$$
\begin{aligned}
& \ddot{\Delta}+2 \frac{\dot{\Delta}}{t}-(2+\omega) R \frac{\Delta}{t^{2}}=\ddot{\lambda}-2(1+\omega) \frac{\dot{\lambda}}{t}-(2+\omega) S \frac{\lambda}{t^{2}}, \\
& \ddot{\lambda}+\frac{\dot{\lambda}}{t}+\left[\frac{n^{2}}{a_{1}^{2}}+S\right] \frac{\lambda}{t^{2}}=-\frac{\dot{\Delta}}{t}+R \frac{\Delta}{t^{2}},
\end{aligned}
$$

where $R=\frac{2 \frac{k}{a_{1}^{2}}-\omega-1}{3+2 \omega}$ and $S=\frac{6 \frac{k}{a_{1}^{2}}+3+\omega}{3+2 \omega}$. These equations may be solved supposing $\Delta=\Delta_{0} t^{r}$ and $\lambda=\lambda_{0} t^{r}, r$ obeying the polynomial equation

$$
\begin{array}{r}
r^{4}+2 r^{3}+\left(\frac{n^{2}}{a_{1}^{2}}+S-(3+\omega) R-(3+2 \omega)\right) r^{2} \\
+\left(\frac{n^{2}}{a_{1}^{2}}-(1+\omega) S+(3+2 \omega) R\right) r-(2+\omega) R \frac{n^{2}}{a_{1}^{2}}=0
\end{array}
$$


This polynomial equation has no simple closed form solution. But, numerical integration reveals the stability of the model in the small wavelength limit. For example, fixing $a_{1}=1$, choosing $\omega=1, k=0$ and $n=1$ we find the roots $r_{1}{ }^{ \pm} \sim-1.16 \pm 2.77 i, r_{2}{ }^{ \pm} \sim 0.16 \pm 0.33 i$, while for $n=100$, keeping unchanged the other parameters, we find $r_{1}{ }^{ \pm} \sim--0.50 \pm 100 i$, $r_{2}{ }^{ \pm} \sim-0.50 \pm 0.97 i$.

\subsection{Gravitational waves}

In this case, the perturbed equation that govern the gravitational waves is

$$
\begin{array}{r}
\ddot{h}+\left(\frac{\dot{\phi}}{\phi}-\frac{\dot{a}}{a}\right) \dot{h}+\left[\frac{n^{2}}{a^{2}}+4 \frac{\dot{a}^{2}}{a^{2}}+2\left(\frac{\omega(\alpha-1)-1}{3+2 \omega}\right)\left(3 \frac{\dot{a}^{2}}{a^{2}}+3 \frac{k}{a^{2}}-\frac{\omega}{2} \frac{\dot{\phi}^{2}}{\phi^{2}}+3 \frac{\dot{a}}{a} \frac{\dot{\phi}}{\phi}\right)\right. \\
\left.-2\left(\frac{1+m+\omega(1+\alpha)}{3+2 \omega}\right) c \phi^{m}\right] h=0 .
\end{array}
$$

The solutions of the above equation are

$$
\begin{aligned}
& \rho=0 \rightarrow h=C_{ \pm} t^{\frac{1}{2}\left(1+A_{1} \pm \sqrt{(1+A)^{2}-4 B-4 \frac{n^{2}}{a_{1}^{2}}}\right.}, \\
& A_{1}=\frac{2+m}{m}, \quad B=\frac{4}{m^{2}}\left(m^{2}-m-2-2 \omega\right), \\
& \alpha=-1 \quad \rightarrow \quad h=C_{ \pm} t^{ \pm \sqrt{-\left(\frac{n^{2}}{a_{1}^{2}}+A_{2}\right)}} \\
& A_{2}=\frac{1}{3+2 \omega}\left[3-8 \omega+4 \omega^{2}-3 \frac{k}{a_{1}{ }^{2}}(1+2 \omega)\right] \text {, } \\
& \alpha=0 \rightarrow h=C_{ \pm} t^{\frac{3 \pm \sqrt{9-4 A_{3}-4 \frac{n^{2}}{a_{1}^{2}}}}{2}} \quad, \quad A_{3}=2-\frac{4 k}{a_{1}{ }^{2}} \\
& \alpha=\frac{1}{3} \rightarrow h=C_{ \pm} t^{2 \pm \sqrt{4 \frac{k}{a_{1}^{2}}-\frac{n^{2}}{a_{1}^{2}}}},
\end{aligned}
$$

where $C_{ \pm}$are integration constants.

Here, the solution for the gravitational waves are also well-behaved and stable.

\subsection{The General Relativity limit}

In general, the Brans-Dicke theory reduces to the General Relativity theory when $\omega \rightarrow \infty$, except in some special cases, for example, when the trace of the momentum-energy tensor is zero [20, 21]. The solutions described above do not have a well-behaved limit when $\omega \rightarrow \infty$. In fact, an inspection of the background equations shows that all solutions become trivial in that limit. We could expect that in this case, the Brans-Dicke field could become constant and the model reduces itself to General Relativity with a cosmological constant. But, the imposition that $a \propto t$ breaks this equivalence.

Concerning the perturbed solutions, they become divergent when $\omega \rightarrow \infty$. This only express the fact that the background scenarios have no sense in this limit. For finite $\omega$, the perturbed solutions exhibit growing and decreasing modes as usual, for scalar and tensorial perturbations. One important feature of the solutions found before is that when $n \rightarrow 0$, all dependence of the solutions on the wavelength of the perturbations is carried

out by the integration constants, which must be fitted correctly in order to reproduce the power spectrum observed today. 


\section{Conclusions}

In spite of the fact that fluids of negative pressure have become crucial to understand many theoretical and observational aspects of modern cosmology, they are plagued with instabilities in the small wavelength limit. These instabilities appear mainly when the barotropic equation of state is such that the strong energy condition is violated. In this paper we have exploited the possibility that these instabilities are due to the hydrodynamical representation. This possibility was suggested by the fact that, while density perturbations are bad behaved in the small wavelength limit, gravitational waves are well behaved in the same limit. Since gravitational waves depend closely on the behaviour of the scale factor but are quite insensitive to the matter representation, the instabilities should be due to the fact that in the hydrodynamical representation, the equation of state is fixed for all wavelength, while in a more fundamental approach it could depend on the scale of the perturbation.

As a matter of fact, this possibility was first suggested in [10], and it has been studied under certains conditions in [18]. In reference [10], this problem has been briefly treated in the realm of minimal scalar-tensor model which was intended to cope with the dark matter problem through the introduction of a scalar field with a convenient potential. However, if we are interested in a field that can drive the accelerated expansion of the Universe, as it is the case in this work, the energy of the scalar field should have a smooth distribution, since it should not be present in the local clusters. Such smooth distribution can be obtained considering that the pressure associated to this field is negative, such that it does not clumpsy in large scale; but in order to avoid instabilities at small scales, the effective equation of state should become positive in this limit, and small fluctuations in this field should oscillate like an accoustic wave.

In the present work, we have verified that, when a fluid of positive pressure can mimic a scalar-tensor model, both formulations are essentially similar in the background and perturbative level. However, when we consider a fluid of negative pressure, the equivalence exists only at the background level: at perturbative level, the models behave in a complete different way. In particular, there is agreement between both representations only in the large wavelength limit: for small wavelengths, the hydrodynamical model is unstable, while the corresponding scalar field representation exhibits accoustic oscillations. Hence, in situations where negative pressures are concerned, a field representation leads to a much more complete scenario, being closer to a realistic model.

This can be understood by remembering that when we pass from a hydrodynamical representation to a fundamental one based for example on scalar fields, we change a relativistic Euler's type equation to a Klein-Gordon equation: the sign of the laplacian operator in these equations are the same only when the hydrodynamical pressure is positive; otherwise, it changes sign and, in the perfect fluid model, accoustic modes give place to exponential modes, resulting in the presence of instabilities. In the long wavelength limit the laplacian operator can be neglected and the results agree in both representation.

We must notice, however, that the scalar-tensor model that corresponds to a given perfect fluid results is quite model dependent. For example, the one fluid model gives a potential that is different from that of a two-fluid models. It would be interesting to employ in a two fluid model, which is closer to a realistic situation, the potential obtained in the one fluid model, in spite of the great complexity of the equations. We can speculate if the resulting effective equation of state evolves in a quite similar way as the 
usual quintessence models.

We have extended this study for the case where the cosmic string fluid mimic a variable cosmological constant model, derived from a non-minimal coupled scalar field with a potential. The conclusions are basically the same as before. But, we must stress that, in this case, there is a Brans-Dicke type coupling parameter $\omega$ that does not recover the known General Relativity solutions when $\omega \rightarrow \infty$. Moreover, the perturbative behaviour may become unstable in this limit, for any scale. But, in general, for finite values of $\omega$ the perturbations do not exhibit either the instabilities that are present in the corresponding hydrodynamical model.

Finally, the fact that both approaches give the same behaviour for the long wavelength limit even if the pressure is negative, implies that the predictions for the power spectrum of the anisotropy of the CMBR for small values of the multipolar expansion parameter $l$, that is, for very large structures, is not spoiled by the employment of the hydrodynamical representation. However, for large values of $l$, that is, small angular separation, the employment of a field representation, mainly when negative pressures are involved, seems crucial.

\section{Acknowledgements}

We thank Nelson Pinto Neto for his suggestions and for many enlightfull discussions. We acknowledge also the financial support from CNPq and CAPES (Brazil).

\section{References}

[1] S. Blau and A.H. Guth, in $\mathbf{3 0 0}$ years of gravitation, edited by S. Hawking and W. Israel, Cambridge University Press, Cambrdige (1987);

[2] S. Perlmutter et al, Nature 391, 51(1998);

[3] A.G. Riess et al, High-z supernovae search team, astro-ph/9805201;

[4] M. Kamionkowski and N. Toumbas, Phys. Rev. Lett.77, 587(1999);

[5] R.L. Davis, Phys. Rev. D35, 3705(1987);

[6] P.J. Stelmach, Horizon problem in a closed Universe dominated by fluid with negative pressure, astro-ph/9810448;

[7] I. Waga and A.P.M.R. Miceli, Phys. Rev. D59, 103507(1999), astro-ph/9811460;

[8] R.R. Caldwell, R. Dave and P.J. Steinhardt, Phys. Rev. Lett. 80, 1582(1998);

[9] I. Zlatev , I. Wang and P.J. Stelmach, Phys. Rev. Lett.82, 896(1999), astroph/9807002;

[10] B. Ratra and P.J.E. Peebles, Phys. Rev. D37, 3406(1988);

[11] P. Brax and J. Martin, Quintessence and supergravity, astro-ph/99050040; 
[12] J.C. Fabris and J. Martin, Phys. Rev. D55, 5205(1997);

[13] J.C. Fabris and S.V.B. Gonçalves, Phys. Rev. D56, 6128(1997);

[14] J.C. Fabris and S.V.B. Gonçalves, Mon.Not.R.Astron.Soc. 306, 679-683(1999);

[15] E.M.Lifschitz and I. Khalatnikov, Adv. Phys. 12, 185(1963);

[16] S. Weinberg, Cosmology and Gravitation, Wiley, New York(1972);

[17] J.C. Fabris and J. Tossa, Grav\&Cosm. 3, 165(1997);

[18] L.P. Grishchuk, Phys. Rev. D50, 7154(1993);

[19] L.O.Pimentel and L.M. Diaz-Rivera, Int. J. Mod. Phys. A14, 1523(1999), grqc/9807016;

[20] C. Romero and A. Barros, Phys. Lett. A137, 243(1993);

[21] V. Faraoni, Phys. Lett. A245, 26(1998). 